\title{
Retrospective Database Analysis Evaluating the Clinical Outcomes of Changing Treatment of People with Type 2 Diabetes Mellitus (T2DM) from Other DPP-4 Inhibitor Therapy to Alogliptin in a Primary Care Setting
}

\author{
W. David Strain (D) - Phil McEwan · Heena Howitt $\cdot$ Simon Meadowcroft
}

Received: May 3, 2019 / Published online: July 1, 2019

(C) The Author(s) 2019

\begin{abstract}
Introduction: Although some differences between individual dipeptidyl peptidase-4 (DPP-4) inhibitors may exist, the National Institute for Health and Clinical Excellence (NICE) have recommended that 'prescribers should be encouraged to select the individual DPP-4 inhibitor with the lowest acquisition cost available to them, where all other factors are equal'. We aimed to determine whether or not 'within class' switching to alogliptin, the DPP-4 inhibitor with lowest acquisition cost, is a clinically appropriate strategy.
\end{abstract}

Enhanced Digital Features To view enhanced digital features for this article go to https://doi.org/10.6084/ m9.figshare.8313392.

Electronic Supplementary Material The online version of this article (https://doi.org/10.1007/s13300019-0662-y) contains supplementary material, which is available to authorized users.

W. D. Strain $(\bowtie)$

Diabetes and Vascular Medicine Research, University of Exeter Medical School, Exeter, UK e-mail: d.strain@exeter.ac.uk

P. McEwan

Health Economics and Outcomes Research Ltd, Cardiff Gate Business Park, Cardiff, UK

H. Howitt · S. Meadowcroft

Takeda UK Ltd, Glory Park, Wooburn Green, Bucks, UK
Methods: This study evaluated people with type 2 diabetes taking DPP-4 inhibitor therapy in addition to at least one other diabetes therapy. Primary care records were reviewed from six clinical commissioning groups (CCGs). For people who had been switched from other DPP4 inhibitors to alogliptin, an assessment of the impact of switch on both absolute haemoglobin A1c (HbA1c) levels and on HbA1c trajectory was undertaken. Persistence on alogliptin and the need for therapy intensification was also assessed.

Results: Overall, 865 people with diabetes met the eligibility criteria for the study. There was no significant difference between pre- and postswitch mean HbA1c level [8.44\% (SD 1.52\%) vs $8.42 \%(1.62 \%), p=0.6]$. Similarly, for patients where there was sufficient data to assess the impact of switching on HbA1c trajectory $(n=319)$ minimal impact was identified (actual HbA1c at 3 months $8.33 \%$ vs projected $8.31 \%$ ). The majority of people with diabetes $(80.76 \%)$ remained on alogliptin treatment at 6 months and only $4.54 \%$ required additional diabetes therapies. Switching to alogliptin resulted in a median saving of $£ 7.24$ per patient-month.

Conclusion: Switching United Kingdom (UK) primary care patients from other DPP-4 inhibitors to alogliptin did not result in a statistically significant or clinically meaningful change in HbA1c level and few required the addition of further diabetes therapies, suggesting that therapy change or intensification was not 
considered necessary in most patients who were switched to alogliptin.

Trial Registration: ENCePP clinical trial registration number EUPAS29153.

Funding: Takeda UK Ltd.

Keywords: Clinical effectiveness; DPP-4 inhibitors; Health economics; Real-world evidence

\section{INTRODUCTION}

The management of type 2 diabetes (T2DM) is becoming increasingly complex. Globally, around 1 in 11 people have diabetes; 90\% of them have T2DM [1]. In the UK, approximately 3.7 million people have been diagnosed with diabetes as of November 2017 [2]. Initial management may involve lifestyle and dietary modifications only, but progressive intensification of pharmacological therapy-usually starting with metformin as first-line treatment-is recommended if adequate glycaemic control is not achieved [3]. Although there is growing evidence for the use of glucagon-like peptide 1 (GLP-1) analogues and sodium-glucose cotransporter-2 (SGLT-2) inhibitors in people with proven atherosclerotic disease or heart failure, in early diabetes the emphasis remains on controlling hyperglycaemia. In early disease, dipeptidyl peptidase-4 (DPP-4) inhibitors are seen as a good option after metformin in primary care as they are perceived as an oral therapy with a reassuring safety profile and with a low risk of hypoglycaemia, as well as being neutral in terms of body weight. Within the class, it has been suggested that there may be some differences in efficacy on the basis of the individual registration studies. There are no head-to-head studies; however, after adjustment for baseline glycaemic control, the impact on HbA1c was broadly consistent across the registration studies [4-8], and the outcomes of the cardiovascular safety trials demonstrated very similar outcomes in people with established cardiovascular disease [9-11]. Therefore, the National Institute for Health and Clinical Excellence (NICE) guidelines state that 'recommendations should consistently refer to DPP-4 inhibitors as a class' and recommend 'that prescribers should be encouraged to select the individual DPP-4 inhibitor with the lowest acquisition cost available to them, where all other factors are equal'. However, given the inconclusive and somewhat limited evidence available at the time of guideline development, NICE considered the interchangeability of DPP4 inhibitors to be uncertain [3].

Some local clinical commissioning groups (CCGs) have recommended implementing a within-class therapy switch to the least costly DPP-4 inhibitor, alogliptin, in people with T2DM. Other CCGs have delayed making wholesale switches as there is some uncertainty around the interchangeability of DPP-4 inhibitors; it is unclear if people switched from one DPP-4 inhibitor to another in routine clinical practice for cost reasons maintain adequate glycaemic control. This study aimed to address this uncertainty by collecting real-world evidence regarding clinical effectiveness results of this cost-driven treatment switch.

\section{METHODS}

We performed a retrospective, observational cohort study which was based on anonymised Read code data routinely captured in primary care systems, extracted for all people with T2DM that were moved to alogliptin from an alternative DPP-4 inhibitor within six CCG localities (Newbury and District, South Reading, North and West Reading, Wokingham, Great Yarmouth and Waverley, and Mid Essex). Switch programs to alogliptin from alternative DPP-4 inhibitors at the discretion of the individual general practitioner took place in all these CCG areas. Anonymised data was extracted from the Electronic Care Leading to Improved Patient Safety and Empowerment (ECLIPSE) computer system which accesses individual patient records, provides prescribers with prescribing safety alerts, information on cost and availability of medications and cost of referrals to secondary care specialists, as well as data pertaining to hospital admissions and outpatient visits. This analysis was performed with the approval of the Caldicott Guardians 
from the respective CCGs, the UK Confidentiality Advisory Group and the South West Regional Ethics Committee (approval number 17/SW/001) in accordance with the principles of the Helsinki Declaration of 1964 and its further amendments.

\section{Eligibility}

Adults (aged $\geq 18$ years) with diabetes and a complete set of baseline characteristics recorded in the database that were switched to alogliptin within the licensed indication [8], from an alternative DPP-4 inhibitor and had at least one HbA1c value prior to the switch (index date) and one post-index HbA1c recorded, with the post-index HbA1c assessment taking place at least 76 days ( 2.5 months) post index-date, were eligible for inclusion. People switched from existing DPP-4 inhibitor monotherapy were excluded from the main analyses reported in this paper, since alogliptin is not licensed for use as monotherapy in the UK.

\section{Primary Endpoint}

The primary objective of this study was to retrospectively estimate the clinical impact of switching people with T2DM from their current DPP-4 inhibitor to alogliptin based on two coprimary endpoints: (1) change from pre- to post-switch HbA1c level and (2) deviation of post-switch HbA1c from HbA1c trajectory, where projected $\mathrm{HbA1c}$ was derived from a statistical model including more than one assessment of pre-switch HbA1c (in order to generate a trajectory) and control covariates.

To maximise the number of people eligible, the first available post-index reading taken at least 76 days after the switch to alogliptin was used. For the HbA1c trajectory analysis, at least two HbA1c records during the pre-index period were required, with the first result at least 90 days after the original DPP-4 inhibitor initiation and a later result at least 60 days after the first result, to allow a reasonable estimate of preindex HbA1c trajectory.

\section{Secondary Endpoints}

If the primary analysis demonstrated that the switch to alogliptin was not associated with a detrimental shift in glycaemic control, we would then go on to determine the persistence of alogliptin therapy at 6 months, the proportion of people switched back to an alternative DPP-4 inhibitor within 6 months, and the percentage of switched people who received further intensification of their diabetes therapy within 6 months of the switch. Finally, we would perform a cost-effectiveness analysis to determine the potential impact of the switch on drug acquisition cost compared to the potential cost of facilitating the switch.

\section{Sensitivity Analysis}

A sensitivity analysis was performed, excluding people with very poor glycaemic control, evidenced by their last pre-switch HbA1c reading (collected within 90 days of the switch) exceeding $9 \% \quad(75 \mathrm{mmol} / \mathrm{mol})$ or $10 \%$ $(86 \mathrm{mmol} / \mathrm{mol})$. All other aspects remained the same as for the main analysis. Finally, an analysis would be performed stratified by baseline HbA1c in $0.5 \%(5.5 \mathrm{mmol} / \mathrm{mol})$ silos.

\section{Statistical Analysis}

Outcomes of interest were evaluated using descriptive statistics, including mean, standard deviation (SD), median, interquartile range (IQR), minimum and maximum, 95\% confidence interval (CI) and frequency of missing data for continuous variables, and frequency and percentage of the sample population for categorical variables. All statistical analyses were performed using $\mathrm{R}$ version 3.4.2.

Differences between pre- and post-index HbA1c measurements were compared using a paired $t$ test. Adjustments were made for CCG locality, patient age and gender. A generalised linear mixed effects model was used to assess changes in HbA1c trajectory as a function of switching to alogliptin (second co-primary outcome), with HbA1c level as the dependent variable, time (split into pre- and post-index 
periods) as a fixed effects variable and CCG as a random effects variable. Baseline characteristics were assessed as potential explanatory variables. An interaction term between period (pre- vs post-index) and any change in concomitant therapy was assessed. Data are presented as coefficient estimates and their associated 95\% CIs.

\section{RESULTS}

Data were extracted for 1085 people with diabetes who had undergone a switch from another DPP-4 inhibitor to alogliptin, within its licensed indication. Of these, 220 did not have appropriate $\mathrm{HbA} 1 \mathrm{c}$ results available either prior to or within 6 months after the switch; therefore it was not possible to include these people in the primary analysis. The characteristics at the index point (day of switch) of the remaining $865(63.7 \%$ male) are shown in Table 1 . They were a typical primary care population with T2DM, with a mean HbA1c of $68.8 \mathrm{mmol} / \mathrm{mol}$ $(8.44 \%)$, blood pressure of $133 / 74$ and total cholesterol of $4.1 \mathrm{mmol} / \mathrm{L}$. Concomitant medication use was predominantly with metformin $(39.08 \%)$, sulphonylurea $(13.41 \%)$ or a combination of the two (45.43\%). As expected, a minority had proven atherosclerotic disease $(0.2 \%$ with cardiovascular disease, $3.1 \%$ with peripheral vascular disease and $6.0 \%$ with prior stroke). The switch to alogliptin did not have any material impact on HbA1c with the postswitch mean value being $68.6 \mathrm{mmol} / \mathrm{mol}$ $(8.42 \%)$. Data on prior DPP-4 inhibitor therapy was available for 684 of 865 people (79.08\%). Time elapsed between the final prescription of the pre-switch DPP-4 inhibitor and index date varied substantially between people (median 63 days, IQR 30-272 days, min 1 day, max 2294 days), suggesting significant non-adherence. We therefore restricted this analysis to those who had a pre-switch DPP-4 inhibitor prescribed within 90 days prior to switch to alogliptin $(n=386)$. Among these patients, HbA1c did not change significantly following the switch $[0.10 \%(95 \%$ CI -0.03 to 0.23$)$, $p=0.14$; Fig. 1]. Neither previous DPP-4 inhibitor prescription nor concomitant medication use had any clinically relevant impact on the effect of the switch to alogliptin on glycaemic control.

For the co-primary outcome examining the impact of the switch on the trajectory of HbA1c, sufficient HbA1c values were available for 319 people, with the principle reason for non-inclusion being an absence of two HbA1c values at least 60 days apart prior to the switch. There were no significant or clinically relevant differences between those included in this analysis and those included in the primary endpoint analysis $(n=865) \quad$ (Supplementary Table 1$)$. When the trajectory models were generated, significant predictors of a steeper (rising) HbA1c trajectory were rising eGFR $(p=0.006)$, rising total cholesterol $(p<0.0001)$ and increasing number of diabetes therapies $(p<0.0001)$. Total number of non-diabetes medications did not have any impact on the trajectory of glycaemic control. Interestingly history of a recent stroke was associated with an improving HbA1c $(p=0.008)$, but this was not replicated with peripheral vascular disease, cardiovascular disease, nephropathy or retinopathy. Performing a switch to alogliptin had no significant impact on the trajectory of HbA1c, with the actual mean value at 3 months being $8.33 \%$ vs projected $\mathrm{HbA} 1 \mathrm{c}$ of $8.31 \%$.

\section{Sensitivity Analyses}

Excluding participants with a baseline HbA1c above $9 \%$ and above $10 \%$ (in whom it may have been more appropriate to escalate therapy rather than performing a simple switch) made little difference to the change in HbA1c after the switch [- $0.02 \%$ (95\% CI -0.10 to $0.10 \%]$ and $0.07 \%$ [ -0.03 to $0.17 \%$ ] for baseline of $\leq 10 \%$ and $\leq 9 \%$, respectively).

When the impact of switch stratified by baseline HbA1c was explored, there was a trend that those who started with the best glycaemic control had a modest deterioration in control, whereas those with the poorest control saw a small improvement in control (Fig. 2). This interaction, however, did not reach statistical significance. 
Table 1 Characteristics of the patient populations analysed

\begin{tabular}{lcc}
\hline Variable & All $(\boldsymbol{n}=\mathbf{8 6 5})$ & \\
\cline { 2 - 3 } & People with missing data, $\boldsymbol{n}$ (\%) & Mean (SD) or $\boldsymbol{n}$ (\%) \\
\hline Change in HbAlc (percentage points) & $0(0.00 \%)$ & $-0.02(1.38)$ \\
HbAlc pre-switch (\%) & $0(0.00 \%)$ & $8.44(1.52)$ \\
Weight $(\mathrm{kg})$ & $4(0.46 \%)$ & $90.13(19.53)$ \\
Height $(\mathrm{cm})$ & $97(11.21 \%)$ & $169.57(9.59)$ \\
Systolic blood pressure (mmHg) & $0(0.00 \%)$ & $132.91(14.22)$ \\
Diastolic blood pressure (mmHg) & $0(0.00 \%)$ & $74.19(9.00)$ \\
eGFR (ml/min/1.73 m $\left.{ }^{2}\right)$ & $4(0.46 \%)$ & $64.64(15.74)$ \\
Cholesterol: total (mmol/L) & $0(0.00 \%)$ & $4.13(0.99)$ \\
Cholesterol: HDL (mmol/L) & $120(13.87 \%)$ & $1.15(0.30)$ \\
Cholesterol: LDL (mmol/L) & $283(32.72 \%)$ & $2.27(2.14)$ \\
Age at switch (years) & $0(0.00 \%)$ & $64.35(11.19)$ \\
History of CVD & $0(0.00 \%)$ & $2(0.23 \%)$ \\
History of PVD & $0(0.00 \%)$ & $27(3.12 \%)$ \\
History of stroke & $0(0.00 \%)$ & $52(6.01 \%)$ \\
History of CKD & $0(0.00 \%)$ & $138(15.95 \%)$ \\
History of diabetic retinopathy & $0(0.00 \%)$ & $47(5.43 \%)$ \\
History of diabetic mononeuropathy & $0(0.00 \%)$ & $1(0.12 \%)$ \\
Male gender & $0(0.00 \%)$ & $551(63.70 \%)$ \\
\hline
\end{tabular}

CKD chronic kidney disease, $C V D$ cardiovascular disease, $D B P$ diastolic blood pressure, HbAlc glycated haemoglobin, $e G F R$ estimated glomerular filtration rate, $H D L$ high-density lipoprotein, $L D L$ low-density lipoprotein, $P V D$ peripheral vascular disease, $S B P$ systolic blood pressure, $S D$ standard deviation

\section{Secondary Analyses}

Out of 865 people in the primary analysis, 705 $(80.76 \%)$ continued alogliptin treatment at 6 months following the switch from another DPP-4 inhibitor. There was a preponderance of men who persisted on alogliptin $(83.7 \%$ vs $75.6 \%$ for male vs female, respectively). Otherwise there were no identified predictors of those most likely to persist on alogliptin to 6 months. Of the 168 who discontinued alogliptin over the first 6 months, the majority $(n=132$; $78.6 \%)$ were not switched to another DPP-4 inhibitor. Of the 705 who remained on alogliptin, 32 people had additional non-DPP-4 inhibitor therapy added. The most common additions were sulphonylurea $(n=8$ people with diabetes), SGLT-2 inhibitors $(n=8)$ and metformin $(n=7)$.

When the financial impact of switching to alogliptin was considered, sufficient details regarding prescriptions before and after switch were available for 349 people. These were predominantly switched from sitagliptin. The total saving in acquisition costs over 6 months was $£ 15,005.76$, based on acquisition costs stated in the British National Formulary (BNF) [12], representing a $19.9 \%$ saving in total prescribing 


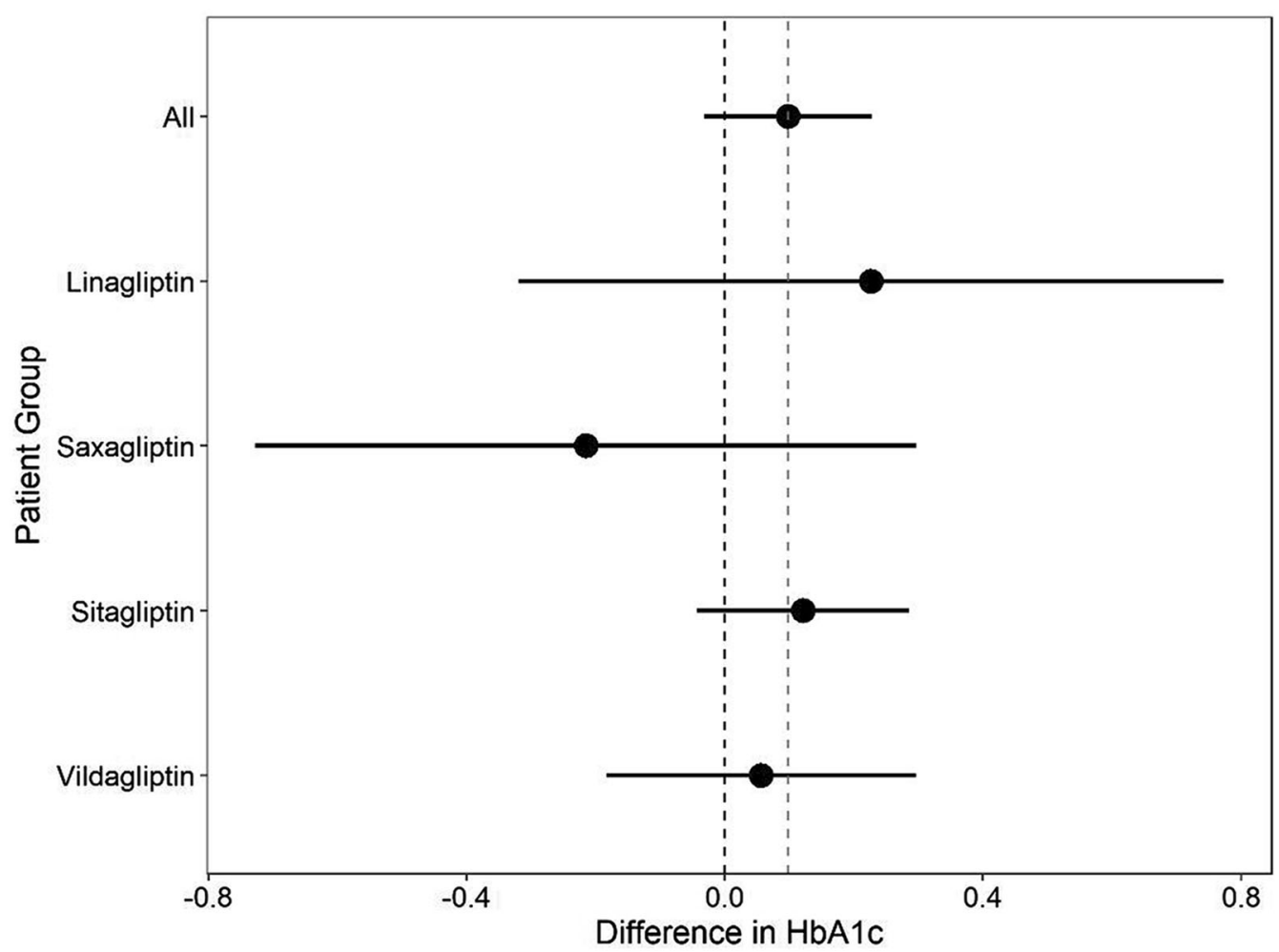

Fig. 1 Change in $\mathrm{HbAlc}$ from most recent pre-switch measurement to first post-switch measurement by preswitch DPP-4 inhibitor prescribed, restricted to last prescription issued within 90 days pre-switch. Data points represent the mean difference in $\mathrm{HbAlc}$ and error bars the $95 \%$ CI. The vertical dotted grey line represents the

cost for these 349 people (median $£ 7.24$ per person per month). There was no change in documented primary care attendances nor in number of investigations requested for the individuals that underwent the switch. However, since duration of consultations is not included in the electronic patient records, it is not possible to estimate any cost implications of service provision in order to perform a switch.

\section{DISCUSSION}

We have explored for the first time the impact of a within-class drug switch program in diabetes, designed to reduce prescribing costs and undertaken in a real-world setting. We demonstrated that glycaemic control did not alter significantly either in absolute terms or from difference between pre- and post-switch $\mathrm{HbAlc}$ level across all patients. The distribution of prior DPP-4 use was sitagliptin $68.4 \%$, vildagliptin $19.7 \%$, linagliptin $6.7 \%$ and saxagliptin $5.1 \%$. CI confidence interval, HbAlc glycated haemoglobin

anticipated trajectory as a result of switching from other DPP-4 inhibitors to alogliptin; however, this switch is associated with a reduction in drug acquisition cost of approximately $20 \%$. The majority $(80 \%)$ of people persisted on alogliptin for at least 6 months and few of these people received further diabetes therapies, suggesting that the switch to alogliptin rarely prompted further therapy change or intensification. Among people who did not maintain the switch to alogliptin, the majority discontinued DPP-4 inhibitors altogether, suggesting that the prescribing physicians rarely considered costlier DPP-4 inhibitors to be more appropriate treatment options than alogliptin. Similar results were obtained in a sensitivity analysis excluding people with very poor glycaemic control (i.e. those with an HbA1c reading $>9 \%$ or $>10 \%$ within 90 days prior to 


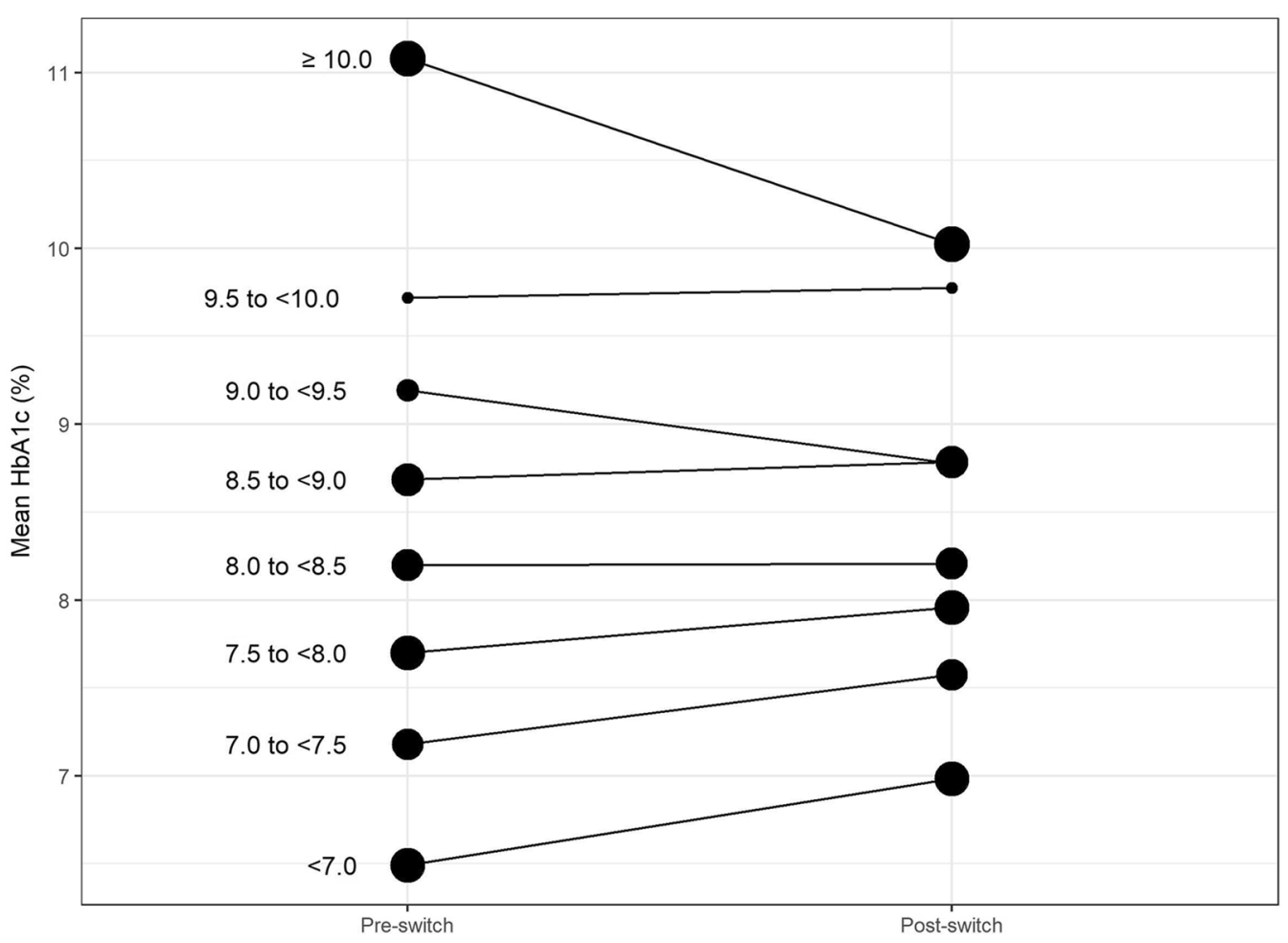

Fig. 2 Change in HbAlc following the switch to alogliptin by baseline HbAlc level. Bullets represent mean HbAlc levels; the size of the bullets corresponds to the size of each patient group. HbAlc glycated haemoglobin

switch), where no statistically significant or clinically meaningful change in HbA1c level following treatment switch was observed.

Projected HBA1c trajectory was significantly affected by people's renal function, total cholesterol level, history of stroke and the number of diabetes therapies prescribed. As may be anticipated, predicted HbA1c was positively associated with renal function (higher eGFR), high total cholesterol levels (a measure of adherence to statin therapy) and a higher number of diabetes therapies. Paradoxically, however, predicted HbA1c was lower in people with a history of stroke, potentially representing the impact of the complications of diabetes on adherence to therapies.

On the basis of previous studies, baseline HbA1c (supplemented by fasting plasma glucose level), and not the type of DPP-4 inhibitor used, is the strongest predictor of response to DPP-4 inhibitor $[13,14]$. The sequential introduction of DPP-4 inhibitors, on a background of the greater understanding of the impact of adverse glycaemic legacy, resulted in the progressive improvement of background glycaemic control over time, with the baseline HbA1c in the first regulatory study being $8.7 \%$ for vildagliptin add-on to metformin, whereas baseline HbA1c levels in subsequent studies were reduced to $8.2 \%$ for saxagliptin, $8.1 \%$ for sitagliptin and $7.9 \%$ for alogliptin. As a result the potential for reduction in glycaemia was evidently reduced with progressive molecule introductions. The paucity of head-to-head trials comparing different DPP-4 inhibitors makes real-world studies that address the issue of their interchangeability particularly valuable. The present study suggests that in routine UK practice, the DPP-4 inhibitor alogliptin is indeed interchangeable with other DPP-4 inhibitors. This finding is in line with the conclusions of a systematic review and network meta-analysis which showed similar efficacy and safety across the DPP-4 inhibitor class [15].

The sensitivity analysis suggested that pretreatment HbA1c predicted response to the 
alogliptin switch, with people experiencing a post-switch decrease in HbA1c having higher baseline HbA1c levels. This may, in part, have been due to people with poor adherence (and thus higher HbA1c) having their motivation to engage with their therapy improved by the active switch from previous DPP-4 inhibitor to the new agent. Indeed, the engagement itself may have improved therapeutic adherence. However, for those at ideal glycaemic control (HbA1c $\leq 53 \mathrm{mmol} / \mathrm{mol}$ ) the switch to alogliptin was associated with a (non-significant) marginal increase in HbA1c. This suggests that while people not at target are not adversely affected, indeed may benefit from, the process of switching to alogliptin, those at optimal control do not benefit, indeed may be adversely affected by the switch. In terms of economic impact, the switch to alogliptin provided a median cost saving of $£ 7.24$ per person per month in those individuals that had collected prescriptions within the 3 months prior to the switch.

\section{Study Limitations}

Based on a large sample of people treated in UK primary care, this study provides real-world evidence on the interchangeability of alogliptin with other DPP-4 inhibitors. However, limitations of the study include a relatively short follow-up (median of 251 days for most analyses) that may have been insufficient to capture the need for therapy escalation in all people. Further, the impacts of different treatment doses were not investigated. We do not believe that this represents a significant limitation, however, since DPP-4 inhibitors (as a class) do not require upwards dose titration, rather they are initiated at optimal dose, then require dose or frequency reduction in those with renal impairment (with the exception of linagliptin).

\section{CONCLUSIONS}

We have demonstrated, for the first time, that the in-class switch from other DPP-4 inhibitors to alogliptin did not compromise clinical outcomes and was generally well tolerated, but potentially reduced DPP-4 inhibitor acquisition costs by approximately $20 \%$. Results from this study suggest that alogliptin is clinically interchangeable with other DPP-4 inhibitors and economically favourable, providing similar glycaemic control at a reduced cost.

Thus, switching people to alogliptin, where clinically appropriate, generates cost-savings with no adverse impact on glycaemic control.

\section{ACKNOWLEDGEMENTS}

W. David Strain would like to acknowledge the support of the NIHR Exeter Clinical Research Facility and the NIHR Collaboration for Leadership in Applied Health Research and Care (CLAHRC) for the South West Peninsula. W. David Strain would like to add that the views expressed in this publication are those of the authors and not necessarily those of the NIHR Exeter Clinical Research Facility, the NHS, the NIHR or the Department of Health in England. All author would also like to acknowledge the staff and patients of the six CCGs that have allowed their data to be used in this anonymous manner.

Funding. The study was sponsored and funded (including the journal's Rapid Service Fee) by Takeda UK Ltd.

Editorial Assistance. We are grateful to Minesh Unadkat for editorial support of the completed text.

Authorship. All named authors meet the International Committee of Medical Journal Editors (ICMJE) criteria for authorship for this article, take responsibility for the integrity of the work as a whole, and have given their approval for this version to be published.

Disclosures. W. David Strain holds research grants from AstraZeneca, Bayer, Novo Nordisk and Novartis, and has received speaker honoraria from AstraZeneca, Bristol Myer Squibb, Merck, Napp, Novartis, Novo Nordisk, and Takeda. Phil McEwan has received substantial 
grant income from Takeda Pharmaceuticals in relation to this study. Heena Howitt is an employee of Takeda Pharmaceuticals. Simon Meadowcroft is an employee of Takeda Pharmaceuticals.

Compliance with Ethics Guidelines. This analysis was performed with the approval of the Caldicott Guardians from the respective CCGs, the UK Confidentiality Advisory Group and the South West Regional Ethics Committee (approval number 17/SW/001) in accordance with the principles of the Helsinki Declaration of 1964 and its further amendments. All authors had full access to all of the data in this study and take complete responsibility for the integrity of the data and accuracy of the data analysis.

Open Access. This article is distributed under the terms of the Creative Commons Attribution-NonCommercial 4.0 International License (http://creativecommons.org/licenses/ by-nc/4.0/), which permits any noncommercial use, distribution, and reproduction in any medium, provided you give appropriate credit to the original author(s) and the source, provide a link to the Creative Commons license, and indicate if changes were made.

\section{REFERENCES}

1. Zheng Y, Ley SH, Hu FB. Global aetiology and epidemiology of type 2 diabetes mellitus and its complications. Nat Rev Endocrinol. 2018;14(2):88-98.

2. Diabetes UK. Diabetes prevalence. 2017. https:// www.diabetes.org.uk/professionals/position-stateme nts-reports/statistics/diabetes-prevalence-2017. Accessed 18 June 2019.

3. National Institute for Health and Care Excellence. Type 2 diabetes in adults: management. NICE guideline NG28. Algorithm for blood glucose lowering therapy in adults with type 2 diabetes. 2015; Updated April 2017. https://www.nice.org.uk/ guidance/ng28/resources/algorithm-for-blood-gluc ose-lowering-therapy-in-adults-with-type-2-diabetes -pdf-2185604173. Accessed 18 June 2019.

4. Merck Sharp and Dohme Ltd. Summary of Product Characteristics: sitagliptin phosphate monohydrate $\left(\right.$ Januvia $\left.^{\circledR}\right)$. Updated August 2018. https://www. medicines.org.uk/emc/product/7887/smpc. Accessed 18 June 2019.

5. Novartis Pharmaceuticals UK Ltd. Summary of Product Characteristics: vildagliptin $\left(\right.$ Galvus $\left.^{\circledR}\right)$. Updated April 2018. https://www.medicines.org. uk/emc/product/6225/smpc. Accessed 18 June 2019.

6. AstraZeneca UK Ltd. Summary of Product Characteristics: saxagliptin hydrochloride $\left(\right.$ Onglyza $\left.{ }^{\circledR}\right)$. Updated August 2018. https://www.medicines.org. uk/emc/product/9368/smpc. Accessed 18 June 2019.

7. Boehringer Ingelheim Ltd. Summary of Product Characteristics: linagliptin $\left(\right.$ Trajenta $\left.^{\circledR}\right)$. Updated March 2016. https://www.medicines.org.uk/emc/ product/4762/smpc. Accessed 18 June 2019.

8. Takeda UK Ltd. Summary of Product Characteristics: alogliptin benzoate $\left(\right.$ Vipidia $\left.^{\circledR}\right)$. Updated May 2018. https://www.medicines.org.uk/emc/product/ 7571/smpc. Accessed 18 June 2019.

9. Green JB, Bethel MA, Armstrong PW, et al. Effect of sitagliptin on cardiovascular outcomes in type 2 diabetes. N Engl J Med. 2015;373(3):232-42.

10. Scirica BM, Bhatt DL, Braunwald E, et al. Saxagliptin and cardiovascular outcomes in patients with type 2 diabetes mellitus. $\mathrm{N}$ Engl J Med. 2013;369(14):1317-26.

11. White WB, Cannon CP, Heller SR, et al. Alogliptin after acute coronary syndrome in patients with type 2 diabetes. N Engl J Med. 2013;369(14):1327-35.

12. Joint Formulary Committee. BNF 74. London: Pharmaceutical Press; 2017.

13. Esposito K, Chiodini P, Maiorino MI, et al. A nomogram to estimate the HbA1c response to different DPP-4 inhibitors in type 2 diabetes: a systematic review and meta-analysis of 98 trials with 24163 patients. BMJ Open. 2015;5(2):e005892.

14. Esposito K, Chiodini P, Capuano A, Maiorino MI, Bellastella G, Giugliano D. Baseline glycemic parameters predict the hemoglobin A1c response to DPP-4 inhibitors: meta-regression analysis of 78 randomized controlled trials with 20,053 patients. Endocrine. 2014;46(1):43-51.

15. Craddy P, Palin HJ, Johnson KI. Comparative effectiveness of dipeptidylpeptidase-4 inhibitors in type 2 diabetes: a systematic review and mixed treatment comparison. Diabetes Ther. 2014;5(1):1-41. 